\title{
Electronic Commerce in China Information Security Management System Strategy Research
}

\author{
Hong $\mathrm{Ji}^{1, \mathrm{a}}$, Shuyuan Zou ${ }^{2, \mathrm{~b}}$ \\ ${ }^{1}$ JiangXi NormalUniversity Science and TechnologyCollege, P.R. China, 330027 \\ ${ }^{2}$ JiangXiNormal University BusinessCollege, P.R. China, 330022 \\ ajxsdjh@vip. sina.com, ${ }^{\text {b } 867879024 @ q q . c o m ~}$
}

\begin{abstract}
Key words:e-commerce; information security; environmental constraints; factors; management system

Abstract.Information security is an important factor in the survival and development of e-commerce, e-commerce throughout the transaction process is always, is the key element of the relationship between the interests of all e-commerce transactions. E-commerce information security to ensure the confidentiality of information is true, is an important part of e-commerce. Based on the analysis of environmental constraints and factors of electronic commerce on the management of information security, e-commerce to our information security management system has been explored.
\end{abstract}

\section{Introduction}

Information security is information network hardware, software, and systems in terms of data protection, free from accidental or malicious destruction of reasons, change, disclosure, continuous and stable operation of the system, the stable operation of the information, the information service is not interrupted. The importance of information security requirements to ensure that information five aspects, namely, the confidentiality of information, authenticity, integrity, unauthorized copying and the parasitic system. Internal information from external threats to information security is a fundamental purpose. Thus, in general, information should be encrypted. To ensure information security, information sources requires authentication, access control, can not have illegal software resides, can not have illegal operation. E-commerce as an important aspect of China's economic development, its information security is directly related to the development of the electronic commerce. As e-commerce aspects of performance of the "weight management, light technology," so our e-commerce in the information security management is the weakest link, analyze the impact factors of e-commerce information security and building management systems is extremely necessary.

\section{Environmental constraints of e-commerce information security management}

Although e-commerce information security in our country has done a preliminary attempt, but the overall situation, its development still has many environmental constraints.

\subsection{Information security risk assessment is not accurate}

Risk assessment is based on risk management, it is first-hand analysis of the existing data network security, e-commerce is one of the most important information security content. The current Chinese enterprises in e-commerce to have a "heavy technology, light management" thinking, so in the process of e-commerce, there is no accurate risk assessment of information security, so that the entire organization in a relatively unsafe framework under e-commerce activities. And thus can not accurately assess the size of the risk and the development of information security policies. This makes it impossible to control the risk of e-commerce, transfer or falls to an acceptable level.

\subsection{Late oversight}

Usually in the development of enterprises in the river, people will focus on the growth and 
development of enterprises, thus ignoring the information on the e-commerce security post supervision, also makes devious criminals always get enterprises or individuals information, then the information to get their so-called "appreciation." Late supervision is not strong, is an important constraint e-commerce information security can not be guaranteed and effectively managed

\subsection{Remedial measures ineffective}

Remedy is an important part of e-commerce information security management, e-commerce companies also rely on a very always factor in development, e-commerce can improve overall information security, security index by remedies. But now when the problem companies deal with information security in e-commerce, are basically in a conservative way, that is, "stop-gap" approach, rather than exploring fundamental upswing, which has not changed the information security of the system itself problem.

\section{Influence Factors of Electronic Commerce Information Security Management}

\subsection{Security Measures}

Security measures to protect e-commerce is the most fundamental information security management. In the era of e-commerce is growing so rapidly, with the increasingly serious problem of information leakage, people are increasingly demanding information security, effective security measures not only to improve the people's trust in the enterprise, but also to ensure that enterprises health e-commerce for sustainable development, but also can improve the efficiency of information security management. It is also a reference to people making factor when e-commerce transactions, will choose a smooth security measures have done quite perfect enterprise. Therefore, the possibility of establishing a series of security measures directly related to e-commerce information security management, tie him down with the growth of e-commerce, thus affecting the development of Chinese enterprises.

\subsection{Monitoring efforts}

Any security measures and the development of perfect security policy are inseparable from the latter part of the monitoring. If monitoring is not in place, even the best strategy is wasted, then the perfect treasure cargo Measures are in vain. For this kind of e-commerce transactions conducted online, which may at any time by external invasion, very easily lead to unsafe information. Monitoring is a pointer to an internal computer monitor pointer, internal computer on the Internet and non-Internet internal behavior and asset management; therefore, it includes Internet Monitoring (Internet behavior monitoring and control of Internet security audit) and internal network monitoring (network behavior monitoring control, software and hardware asset management, data and information security), and some also increased the transparency of data security encryption software deployment, it is a powerful post monitoring is particularly important in e-commerce information security management.

\subsection{Remedies}

The speed of development of electronic commerce compared with the developed countries there are still some gaps, it is inevitable in the e-commerce information security issues will be some loopholes, we need to do is back again and again problems sum up experience, to mention wrong remedial measures to improve the security of our information. An effective remedy not only to solve immediate problems, but also to discover the underlying problems, timely repair and further improve the security of information systems. So a powerful remedy to consolidate strong e-commerce and information security management efforts for the development of e-commerce enterprises to bring the role of icing on the cake. 


\section{Our e-commerce information security management system optimization}

After the above factors and environmental constraints of e-commerce information security management analysis, this paper from the information security policy, security measures, four monitoring and remedial measures to e-commerce information security management system design.

The information security policy selection and implementation of security measures. With improvements in security technology and security products, these security measures should be changed regularly, but to follow the appropriate information security policies. At the same time the organization but also for safety education and training of its staff, and its security responsibilities for different roles depending on the staff. Each staff member should develop individual annual information security program, and according to the plan, the implementation of the security plan at the end of the year.

Pairs of protecting information security system is necessary, but not completely eliminate the risk of information security, it is necessary for the entire information system for real-time monitoring, in order to detect unusual activity. Enterprises can establish information security monitoring system Agent-based, to achieve real-time monitoring information system.

In the case of information systems has been successfully invaded and destroyed, and take appropriate remedial measures, allowing companies to conduct normal business processes, and risk analysis and assessment again to increase or change existing information security measures. In normal operation of information systems, e-commerce information security managers must regularly back up the system.

\section{Conclusion}

E-commerce information security management is an important factor determining whether our enterprise e-health and sustainable development, information security management is a powerful weapon to beat its competitors in the field of competition. Although China's e-commerce information security management aspects of the multifaceted try and get objective results, but the entire e-commerce information security management, there are still many environmental constraints. Factors affecting our e-commerce information security management, the development of information security policy is an important prerequisite for e-commerce information security management, reflecting the business on their own e-commerce information security awareness and risk assessment; security measures e-commerce information security security management; monitoring of e-commerce information security management indispensable aids; remedies are e-commerce information security management of the reserve army. We should be four areas of information security policies, security measures, monitoring efforts to remedy, in the context of relevance, feasibility, design of e-commerce information security management system.

\section{References}

[1]Yao Jia han.Optimization of business structure and website information ecological chain research [D]. Jilin University, 2014.

[2]Wang show. Cloud computing environments Commerce Security Risk Assessment Model [D].Anhui University of Finance and Economics, 2015.

[3]Du Yuehua.Huaneng Power International Electronic Commerce Network Operations Management Innovation and security research [D]. North China Electric Power University, 2015.

[4]Zhang Qi.Based on e-commerce information security evaluation model and method of Vague Set [D].Yunnan University of Finance and Economics, 2013.

[5]Wang Lifeng Electronic Commerce Information Security Management Architecture [J] Network Security Technology and Application, 2015,04: 24-25. 